Sharif University of Technology
Scientia Iranica
SCIENTIA
I RAN ICA
http://scientiairanica.sharif.edu

\title{
Earthquake ground-motion duration estimation using general regression neural network
}

\author{
S. Yaghmaei-Sabegh* \\ Department of Civil Engineering, University of Tabriz, Tabriz, Iran.
}

Received 22 May 2016; received in revised form 4 February 2017; accepted 13 March 2017

\author{
KEYWORDS \\ Ground motion \\ duration; \\ Significant duration; \\ Generalized \\ Regression Neural \\ Network (GRNN); \\ RBF network; \\ Iran.
}

\begin{abstract}
Accurate prediction of earthquake duration could control seismic design of structures. In this paper, a new simple method was developed to estimate such an important parameter by employing Artificial Neural Networks (ANN) capability. A Generalized Regression Neural Network (GRNN) as a special class of RBF networks was implemented in this study to reduce the number of computation steps required for the searching process on sparse datasets. This network with quick-design capability does not need to impose a prescribed form to map the observed data. The independent variables used in the predictive model of this study included earthquake magnitude, distance measure, and site conditions. The designed models were trained using the 950 accelerograms recorded at Iranian plateau. The performance of the proposed approach was compared with predicted results of feedforward backpropagation networks. Analyses show that the designed GRNN performs well in estimating earthquake record duration and can be applied to predict common measures of earthquake ground-motion duration.
\end{abstract}

(C) 2018 Sharif University of Technology. All rights reserved.

\section{Introduction}

Amplitude parameters, such as peak ground acceleration or spectral acceleration, are widely used parameters in most seismic design codes. Nevertheless, the duration of strong motions could enlarge the extent of earthquake damage to structures. According to various studies, seismic response of a structure depends on a structural framing system and earthquake characteristics including amplitude and duration [1-4]. Ground-motion duration significantly affects ductility measure, the amount of cumulative damage incurred by the structures, dissipated energy [5,6], input energy amount, and the level of damage done to a structure. It is noted that the role of duration depends on several

\footnotetext{
*. Fax: +984113344287

E-mail address: s_yaghmaei@tabrizu.ac
}

factors such as the structural model and damage metric used in the analysis [7]. From a geotechnical point of view, duration of input motion is expected to play an instrumental role in evaluating soil's seismic response, liquefaction potential, and lateral spread displacement resulting from soil liquefaction [8-10]. Ground-motion duration has also an imperative role in the assessment of potential losses in future earthquakes as in the HAZUS standardized framework, where duration is explicitly taken into account $[11,12]$.

Several researches, conducted in the past, attempted to obtain the earthquake ground-motion duration based on regional (local) predictive equations. These predictive equations, typically fit into a strong ground-motion data by conventional regression methods, could be developed for typical definitions of the ground-motion duration. Kempton and Stewart [3] proposed significant duration prediction equations based on a random effects regression procedure with respect to different parameters: magnitude, distance 
from site to source, soil site condition, and one more factor that reflects near-field effects. Bommer et al. [2] employed the global database of Next Generation of Attenuation (NGA) to present new updated empirical equations. Recently, Yaghmaei-Sabegh et al. [13] developed a new predictive model by a nonlinear regression analysis based on earthquake ground-motion records obtained in Iran. According to the basic seismological theory, earthquake record duration depends on the complex fracture mechanism on the fault plane and seismic wave radiation characteristics. Thus, utilizing an appropriate physically based representation for such a multi-faceted parameter would be very difficult and may require a deep understanding of different parameters that control ground-motion duration. For this reason, different mathematical functions often in the nonlinear forms have been adopted in the associated literature. To overcome this concern, the purpose of this paper is to develop a simple tool for estimating the duration of earthquake records. To this end, a simple and efficient framework is designed based on high capability of GRNN neural networks.

By increasing the computational power of engineering software, different computer-based searching algorithms, including K-means, Genetic Programming (GP), Artificial Neural Networks (ANNs), and autoregressive integrated moving average (ARIMA), are vastly used in both seismology and earthquake engineering [14-17]. This paper shows that the nonlinear nature and high fitting ability of a special class of networks, named Generalized Regression Neural Networks (GRNN), make it very suitable particularly while different factors influence the prediction results. As an advantage for a GRNN, design-decisions about the layers numbers and unit number of the hidden layers could be removed.

The purpose of this paper is an attempt to establish a suitable platform to compare capabilities of Multi-Layer Feed-Forward (MLFF) and GRNN networks for accurate prediction of ground-motion record duration as a complex problem in seismology. The need for GRNN implementation for the purpose of this study is demonstrated by presenting the prediction results of backpropagation multi-layer feed-forward networks. This paper consists of six different sections. The study of this paper could facilitate a good condition for readers to compare the prediction abilities of multi-layer feed-forward neural network and GRNN models with regard to a complex case study in seismology where training data are limited. Following the introduction, a description of the artificial neural networks applications in seismology and earthquake engineering is provided in Section 2. Theoretical background of implemented network is laid out in this section, too. Section 3 reviews different definitions for ground-motion duration. Results of analysis in training and testing steps are discussed in Section 4. This section also includes the details of the ground-motion records and dataset analysis. The prediction ability of MLFF network as the most popular ANN is evaluated and compared with others in Section 5. Different performance evaluation indices, i.e., root mean square error, correlation coefficients, and efficiency factor between estimated and observed dataset, are used in the analysis. A summary of conclusions drawn in this study is reported in the final section of the current paper.

\section{Artificial neural networks and applications}

Capability of Artificial Neural Network (ANN) to model complex features was used successfully in the past when conventional linear functions were not able to demonstrate high-dimensional inputs. The applications of artificial neural networks as a powerful tool have been evaluated broadly in seismology, earthquake and geotechnical engineering, and geosciences.

McCulloch, a neurobiologist, and Pitts, a statistician [18], initiated the first artificial neuron as a binary threshold unit in 1943. An ANN deduces essential features of biological neurons and their interconnections and takes a different approach to solve a specific problem than that of conventional algorithmic computer, which follows a set of instructions in order to solve the problem. Neurons (or cells) supply parallel processing nature of artificial neural networks to solve a complex problem [19]. Each neuron is responsible for carrying out received impulses from input cells to the other cells. Neural networks are able to learn during training process to achieve the generalization ability, which is useful for future predictions. In this regard, neural networks could be considered as a practical tool for pattern recognition and function approximation applications. Multi-Layer Feed-Forward (MLFF) neural network, referred to as multi-layer perceptron, Kohenen's Self-Organizing Maps (SOM), and RBF networks are different types of neural networks, normally applied to solve such a problem.

In a MLFF network, which is a most popular ANN architecture, feedforward style, typically, is used to form connections among hidden layer neurons with those of input and output layers. The error backpropagation (BP) algorithm as a highly popular algorithm for feedforward networks uses a local gradient to reach a minimum value of prediction error. The MLFF was employed widely in the past researches as a predictor of unknown functional relations for different applications in both seismology and earthquake engineering. Pioneering researches by Dysart and Pulli [20] and Dai and MacBeth [21] were focused on the application of BP-MLFF in regional seismic event classification and identification of seismic arrival types. Application of a back-propagation neural network in system identifica- 
tion (stiffness and damping coefficients) and structural response prediction due to earthquake excitation can be found in the literature [22,23]. Kuzniar et al. [24] implemented capability of MLFF neural networks to construct response spectra based on mining tremors data. Gentili and Bragato [25] proposed a BP-MLFF neural network system to forecast earthquakes location occurred in Italy. Asencio-Corte's et al. [26] evaluated the efficiency of neural networks for the prediction of earthquake sizes according to five databases in Japan. In 2005, in Taiwan, Kern and Ting [27] predicted peak ground acceleration values by considering MLFF neural networks as a soft computing tool. Ahmad et al. applied an artificial neural network to develop attenuation relationships for peak Parameters as Ground Acceleration (PGA) [28]. They considered real earthquake data to demonstrate the accuracy of designed network to model local attenuation characteristics. Arjun and Kumar [29] presented a new application of MLFF neural network to estimate earthquake record duration in Japan. Recently, Liu et al. [30] applied a neural network as a classifier along with wavelet transform to structural damage diagnosis. Alarifi et al. [31] used a feedforward neural network (consisting of 3 layers) to predict earthquakes magnitude in northern Red Sea area. As another application, high ability of neural networks was used for earthquake prediction based on correlated information with earthquake occurrence in the past [32-34]. Panakkat and Adeli [35] proposed a neural network model to provide useful information about events location and time of occurrence for major earthquakes in the California. Despite wide applications of this type of neural networks, there are a number of disadvantages in using BP-MLFF models. The architectural design of MLFF neural networks, including optimal number of neurons in each of hidden layers, is difficult. For lower number of nodes, the network does not obtain the right results. In contrast, increasing the number of units increases the number of weights [36] and, hence, the processing time in training step; sometimes, it is weakens the generalization ability of the network. Typically, it can take a large number of iterations to converge to the preferred values and, consequently, require too much time, particularly when a large-sized network is needed.

The feasibility of using Generalized Regression Neural Networks (GRNN) is examined in this article to predict earthquake ground-motion duration. More information about RBF and GRNN networks is presented in Sections 2.1, and 2.2. Details of the mathematical theory of neural networks were extensively documented in $[37]$.

\subsection{RBF-based models}

A Radial Basis Function network (RBF) learns using a supervised training technique and consists of a single hidden layer associated with basis functions modelling a Gaussian response surface. Indeed, RBF network performs a nonlinear mapping when the data are loaded onto interior configuration of network. The advanced capability of RBF networks in civil engineering and seismological problems has been shown in estimating design parameters [38], identification and control of structures $[39,40]$, prediction of building interference effects [41], earthquake magnitude prediction [42], seismic data inversion problem [43] in stress-strain approximation of plain concrete [44], and, recently, for estimation of earthquake occurrence model [45].

\subsection{GRNN-based model}

A statistical technique called "kernel regression" performs a fundamental role in the proceeding core of GRNN, which will be used for prediction herein. As a main advantage over traditional regression methods, GRNN, like kernel methods in general, does not need to impose a prescribed form to map the observed data. In fact, GRNN model is able to construct an appropriate representation based on probability density function of the input data, facilitating smooth transition amongst different observations [46]. Training time of GRNN which is a memory-based network is short, because the bandwidths of the parameters used in the analysis are simply considered [47]; therefore, the precise setting is not needed [48]. Figure 1 demonstrates a typical architecture of GRNN consisting of four layers. The input and output layers in GRNN are similar to those of most neural networks. However, there is no computational role in the neurons of input layer in this type of network, where data are simply passed to the pattern layer units. Pattern and summation layers are two computational parts of GRNN that complete the structure of GRNN. Neurons are assigned to all of training datasets in the pattern layer to compute the Euclidean distance based on the center-point position of neurons. Finally, the RBF kernel function has been applied to this processing layer. The summation layer contains two neurons: S-summation and D-summation neurons, which compute the sum of weighted and unweighted outputs, respectively. The summation and output layers produce a normalization of output set. as follows:

The predicted target value $(\hat{y})$ can be explained

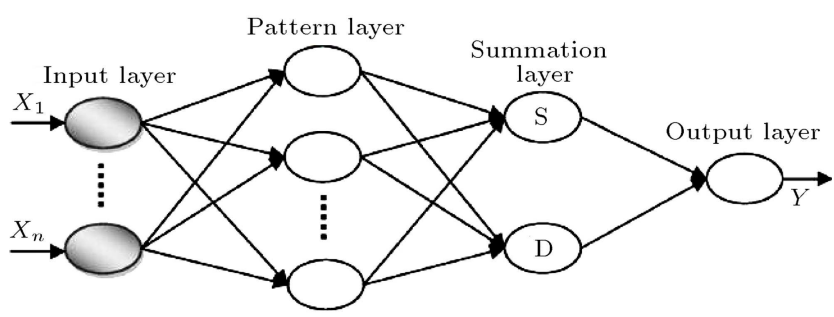

Figure 1. A typical architecture of generalized regression neural network. 


$$
\hat{y}=\frac{\sum_{j=1}^{n} y_{j} \exp \left[-D\left(x_{i}, x_{i j}\right)\right]}{\sum_{j=1}^{n} \exp \left[-D\left(x_{i}, x_{i j}\right)\right]}
$$

where $y_{i}$ is the weight connection between the $i$ th unit in the pattern layer and the summation layer, $n$ is the number of training cases $\left(x_{i j}\right)$, parameter $x_{i}$ is input value for testing cases, and function $D$ is described as follows:

$$
D\left(x_{i}, x_{i j}\right)=\sum_{i=1}^{p} \frac{\left(x_{i}-x_{i j}\right)^{2}}{2 \sigma^{2}}
$$

in which $p$ indicates the unit number of each input vector, $\sigma$ is called "smoothing" or "bandwidth" parameter which affects prediction performance of a GRNN and is frequently calibrated for each model [49]. More information about GRNN model can be found in $[48,50]$. The main features of GRNN in function approximation could be summarized as follows:

i) The simplicity in design where there is no need to define a learning rule;

ii) Anticipating high performance often even based on small amount of data;

iii) Low cost of CPU processing;

iv) Removing the decision-making step on architectural design of network.

It is well known that, similar to an RBF network, GRNN model is not able to extrapolate. For this reason, a GRRN designer should be aware of this important matter to control the range of the selected training data used in the analysis.

A review of the past research works illustrates different applications of GRNN in seismology and earthquake engineering. GRNN was applied to evaluate soil composition based on CPT data by Kurop and Griffin [51]. Hanna et al. [52] used high capacity of GRNN to estimate soil liquefaction potential based on earthquake database of Turkey and Taiwan. In 2011 and as an important procedure in site-specific seismic hazard assessment, a GRRN-based procedure was suggested for site classification [47]. The results of Yaghmaei-Sabegh and Tsang [47] were validated with borehole data that are normally used in the soil classification procedure. Their results revealed high efficiency of GRNN model used for this purpose $[47,53]$.

\section{Overview of different ground-motion duration definitions}

As mentioned before, ground-motion duration may involve many variables from source, path and site effects; consequently, there is no general definition for this complex-multifaceted phenomenon among seismologists. Different typical definitions, presented for earthquake record duration in the past, could be put into three main groups: bracketed, uniform, and significant durations.

The bracketed duration measure $\left(D_{B}\right)$ is the time duration of ground shaking that is defined as the time length from the first and last excursions rather than a precise pre-defined threshold of acceleration [1]. This simple definition is a sensitive measure for the acceleration threshold and can be unstable in some cases [1]. It should be noted that bracketed duration has been used by different researchers, taking into account different levels of acceleration as a threshold $[2,54,55]$.

Uniform duration $\left(D_{U}\right)$ is measured as the sum of numbers of discrete time intervals at the points where acceleration is greater than a pre-defined threshold. It is noted that the sensitivity of this definition is lower compared with that of bracketed duration. Figures 2 to 4 demonstrate the acceleration time history as well as bracketed and uniform durations for a destructive earthquake occurred at Tabas, northeast of Iran (recorded at Deyhook station).

Finally, significant duration $\left(D_{S}\right)$ as an energybased measure describes a continuous time window, which is defined based on two pre-defined Arias inten-

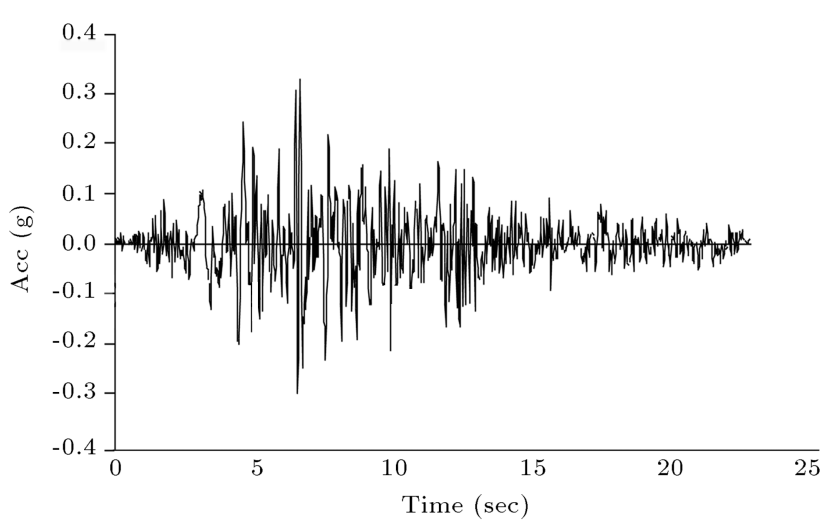

Figure 2. Acceleration time history of 1978 Tabas ground-motion recorded at Deyhook station.

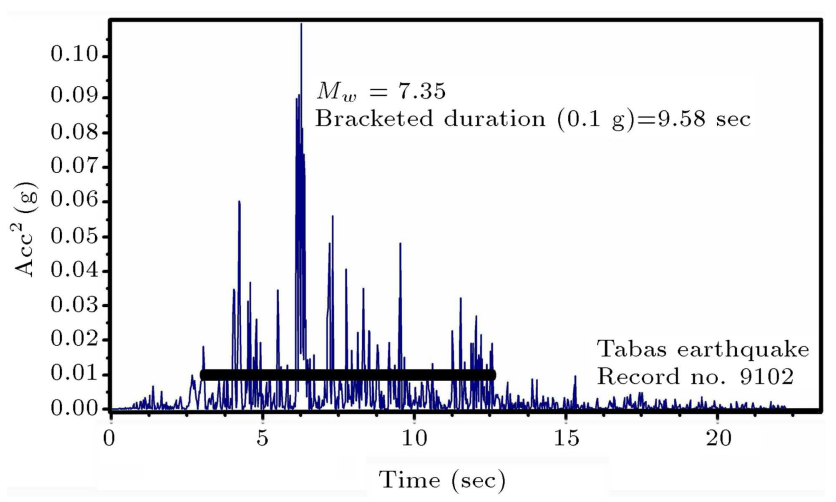

Figure 3. Bracketed duration $\left(D_{B}\right)$ estimated for 1978 Tabas earthquake recorded at Deyhook station $\left(\mathrm{Acc}^{2}\right.$ is the square of the ground acceleration). 


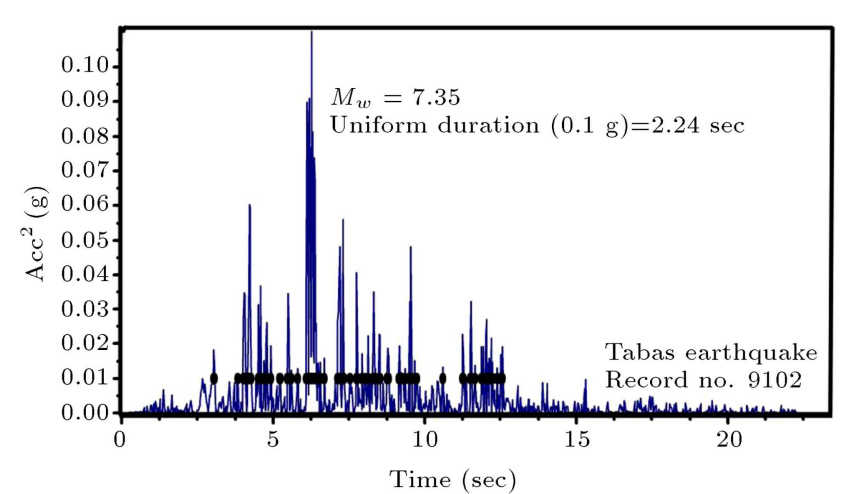

Figure 4. Uniform duration $\left(D_{U}\right)$ estimated for 1978 Tabas earthquake recorded at Deyhook station $\left(\mathrm{Acc}^{2}\right.$ is the square of the ground acceleration).

sity thresholds. The significant duration illustrates the time interval of ground-motion time history when the main part of input energy is imposed on the buildings and is more stable than the bracketed and uniform duration measures. For these reasons, two generic measures of this definition are used in the prediction procedure of this research as the time intervals of Arias Intensity between $5-95 \%$ and $5-75 \%\left(D_{s-5-95 \%}\right.$ and $\left.D_{s-5-75 \%}\right)$, respectively. Figure 5 presents these two common measures of significant duration for the selected record at Deyhook station.

\section{Estimating earthquake ground-motion duration}

\subsection{Model development}

The primary step to design a GRNN model is to provide a suitable large database used in learning and testing process. The ground-motion records applied in this work corresponded to 950 ground-motion records obtained at important earthquakes occurred in Iran, provided by Building and Research Center (BHRC). A review of the historical earthquakes in Iran demonstrates this fact that Iran has been located in one of

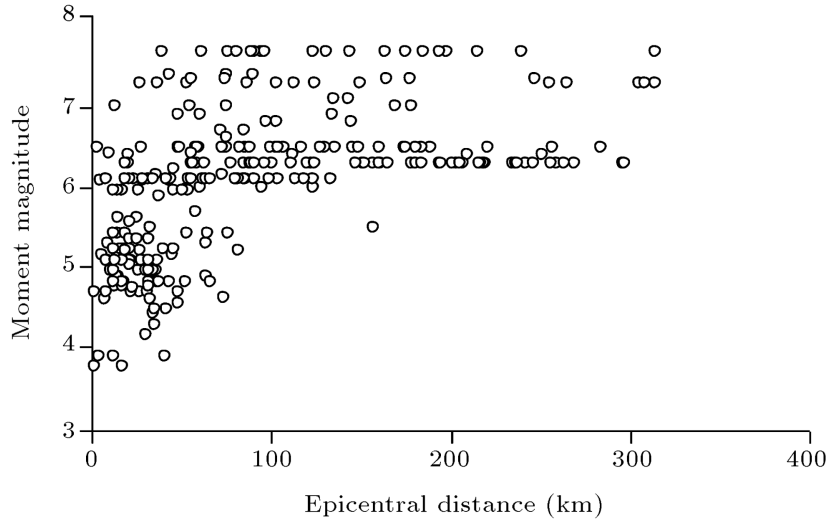

Figure 6. Magnitude versus closest site-source distance of dataset used in this study.

the world's significant seismic belt that has experienced many large events in the past [56].

Updated database of this paper covers the ground-motion duration data, recently used by Yaghmaei-Sabegh et al. [13] to develop a new predictive model in Iran. The overall range for moment magnitude in the dataset is from 3.75 to 7.7 with the closest site-source distance ranging from 1.5 to $370 \mathrm{~km}$. Figures 6 and 7 demonstrate the magnitude of earthquakes used in this study against distance measure along with the location distribution of important earthquakes across the study area, Iran. The total set of 950 values used for the modelling is separated into two data bins. Eighty percent of dataset has been adopted as training set and $20 \%$ of data can best cover testing set of analysis.

In the proposed models, an earthquake groundmotion duration parameter is described as a function of three independent variables: earthquake size, distance from source to site, and soil type, i.e., $D_{s}=$ $f(M, R, S)$. Two different models have been presented separately for two generic measures of significant duration $\left(D_{s-5-95 \%}\right.$ and $\left.D_{s-5-75 \%}\right)$. The closest sitesource distance is used also as a distance measure

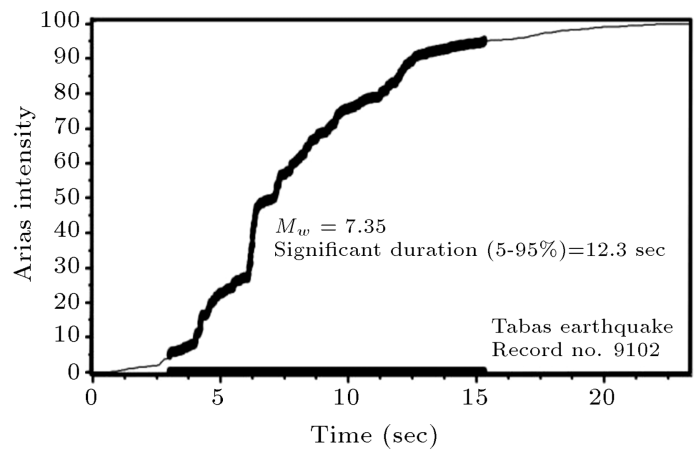

(a)

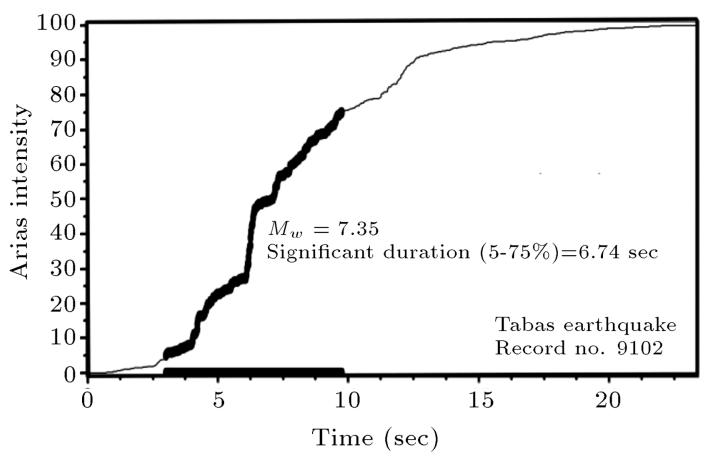

(b)

Figure 5. Significant duration estimated for 1978 Tabas earthquake recorded at Deyhook station $\left(D_{s-5-95 \%}\right.$ and $D_{s-5-75 \%)}[13]$. 


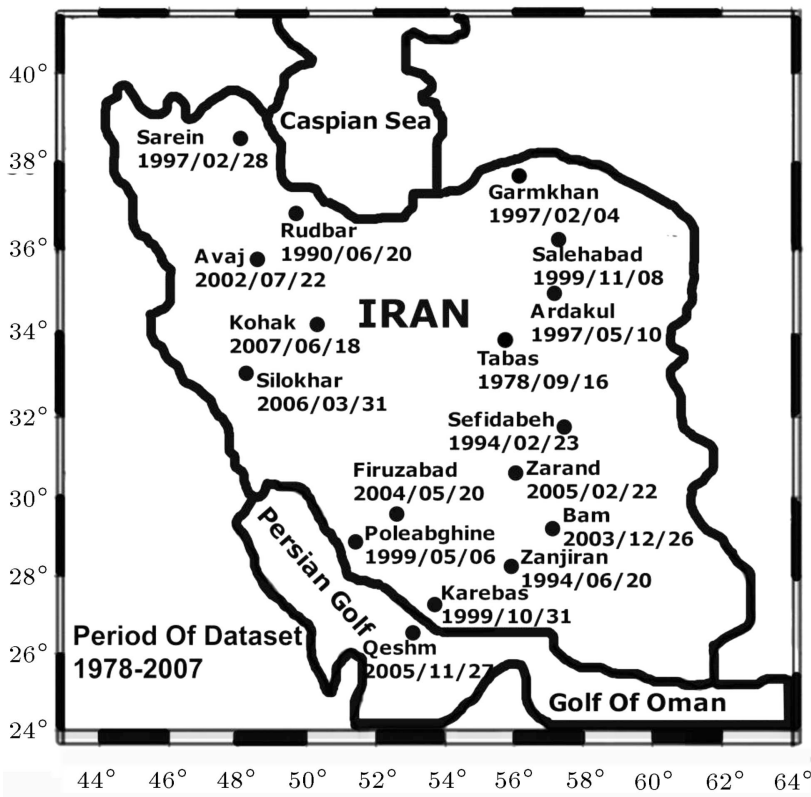

Figure 7. Location distribution of major earthquakes used in this study for the prediction of earthquake-ground motion duration [13].

herein. Site effect in the proposed duration model is considered to be based on soil classification scheme adopted in the Iranian seismic design code (Standard 2800). Four soil classes have been defined in this code based on average shear wave velocity, which is compatible with site classes in 2003 NEHRP [57]. Dummy variables 0 and 1 are used simply in the prediction process for rock and soil sites, respectively.

\subsection{Results}

The only factor that needs to be selected to design GRNN model is the smoothing parameter that affects the predicted value of the designed neural network. Generally, smoother prediction would be expected when the smoothing parameter is larger. In addition, generalization capability of designed network decreases for small value of smoothing parameter, which is not an enviable quality for future predictions. Therefore, the suitable value of this parameter, which is often experimentally determined, will play a significant role in design implementation of this type of networks. In this study, different values of smoothing parameter ranging from 0 to 1 have been examined; finally, based on prediction performance, the value of $\sigma=0.7$ has been intended through a calibration process. It is worth noting that choosing an appropriate value for smoothing parameter will be more important if the number of observation may be small enough to predict a complex phenomenon

The efficiency and robustness of designed networks have been checked based on three statistical indices named root mean square error, correlation coefficients, and efficiency factor. These indices are

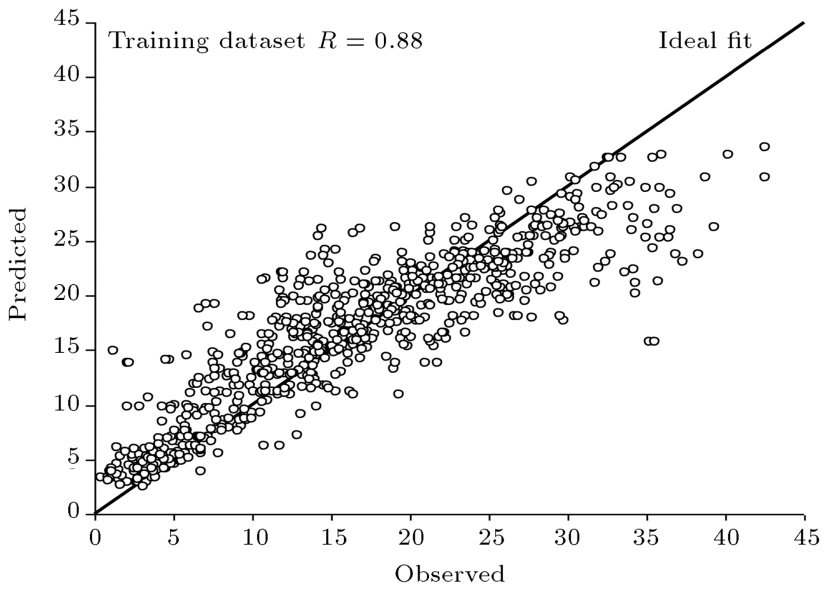

Figure 8. Predicted values of significant duration $D_{s-5-95 \%}$ versus observed values in training set.

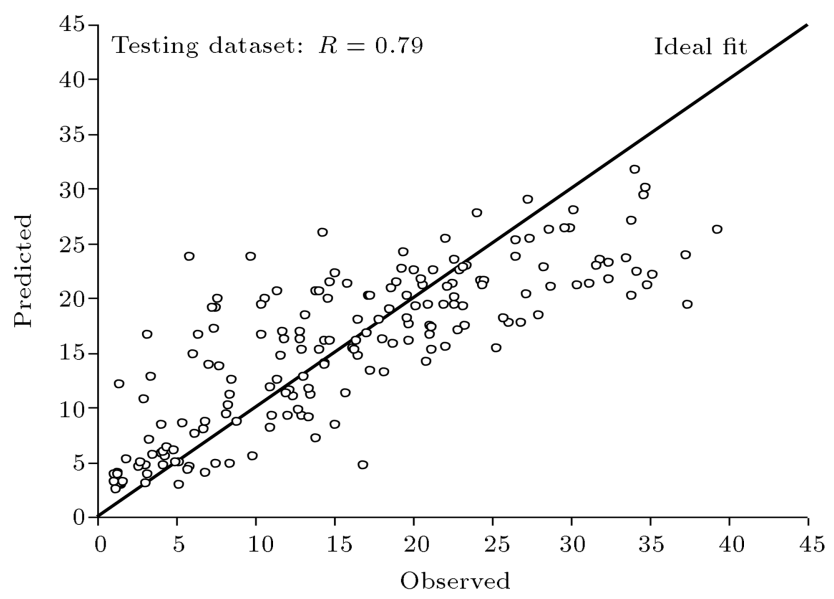

Figure 9. Predicted values of significant duration $D_{s-5-95 \%}$ versus observed values in testing set.

defined as follows:

$$
\begin{aligned}
& \mathrm{RMSE}=\sqrt{\frac{\sum_{i=1}^{N}\left(X_{i}-Y_{i}\right)^{2}}{N}}, \\
& R=\frac{\sum_{i=1}^{N}\left(X_{i}-\bar{X}\right)\left(Y_{i}-\bar{Y}\right)}{\sqrt{\sum_{i=1}^{N}\left(X_{i}-\bar{X}\right)^{2} \sum_{i=1}^{N}\left(Y_{i}-\bar{Y}\right)^{2}}}, \\
& \mathrm{EF}=\frac{\sum_{i=1}^{N}\left(X_{i}-\bar{X}\right)^{2}-\sum_{i=1}^{N}\left(Y_{i}-\bar{Y}\right)^{2}}{\sum_{i=1}^{N}\left(X_{i}-\bar{X}\right)^{2}} .
\end{aligned}
$$

In these equations, $X_{i}$ and $Y_{i}$ are defined as the observed and predicted values; $\bar{X}$ and $\bar{Y}$ are the mean values of the observed and predicted data, respectively [58]. $N$ is the number of data in dataset analysis. The three well-known indices, used in this study, reflect the degree of fit for the proposed models and could evaluate the ANN output error between the actual and predicted outputs. Figures 8 through 11 illustrate the ability of designed network while presenting scatter 
Table 1. Performance of designed GRNN for predicting $D_{s-5-95 \%}$ and $D_{s-5-75 \%}$.

\begin{tabular}{cccccccc}
\hline \multirow{2}{*}{ Duration } & \multicolumn{3}{c}{ Training set } & & \multicolumn{3}{c}{ Testing set } \\
\cline { 2 - 3 } \cline { 6 - 8 } & $\boldsymbol{R}$ & EF & RMSE (sec) & & $\boldsymbol{R}$ & EF & RMSE (sec) \\
\hline$D_{s-5-95 \%}$ & 0.88 & 0.77 & 4 & & 0.79 & 0.73 & 4.1 \\
$D_{s-5-75 \%}$ & 0.89 & 0.78 & 2.54 & & 0.76 & 0.75 & 2.68 \\
\hline
\end{tabular}

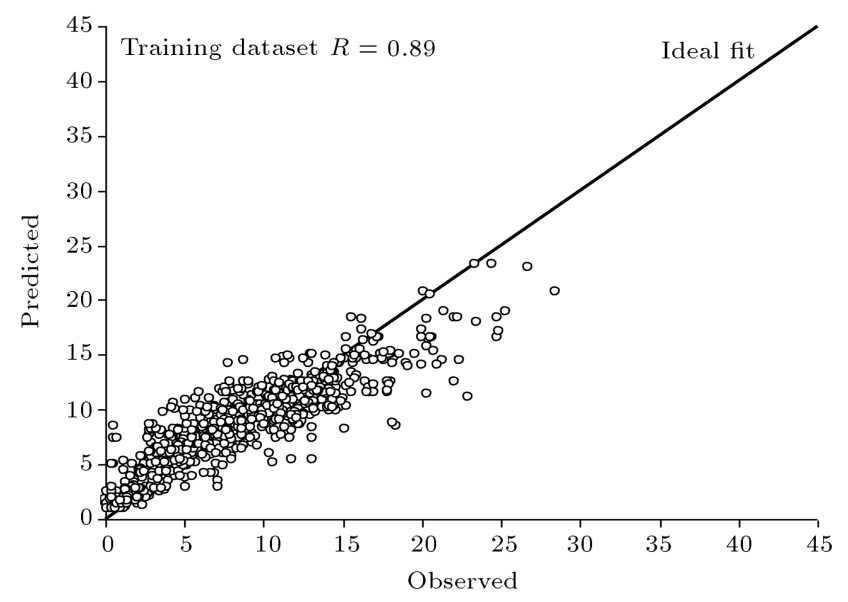

Figure 10. Predicted values of significant duration $D_{s-5-75 \%}$ versus observed values in training set.

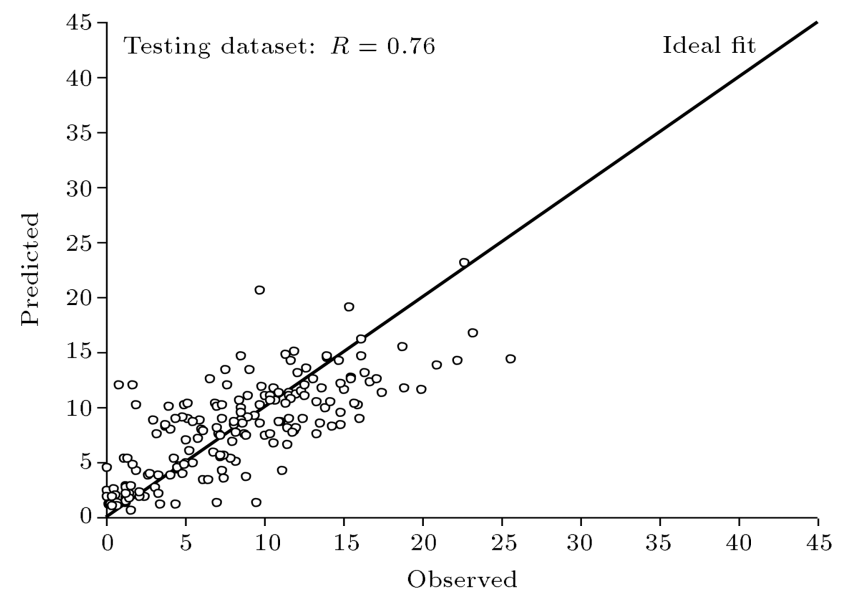

Figure 11. Predicted values of significant duration $D_{s-5-75 \%}$ versus observed values in testing set.

plots of the predicted values against observed values for predictive models of $D_{s-5-95 \%}$ and $D_{s-5-75 \%}$. Observed/estimated $=1$ line is also superimposed on the figures. Table 1 indicates the results of comparisons of GRNN performances for training and testing dataset. It is revealed that the values of the RMSE and EF of $D_{s-5-95 \%}$ and $D_{s-5-75 \%}$ models are close to each other, and the designed GRNN model could predict these two parameters almost with similar accuracy. The total residual as another indicator is employed to evaluate the performance of soft computing predictions by GRNN. Figures 12 and 13 show the residual $\%$ vspace* $0.5 \mathrm{~cm}$ scattering plot for significant dura-

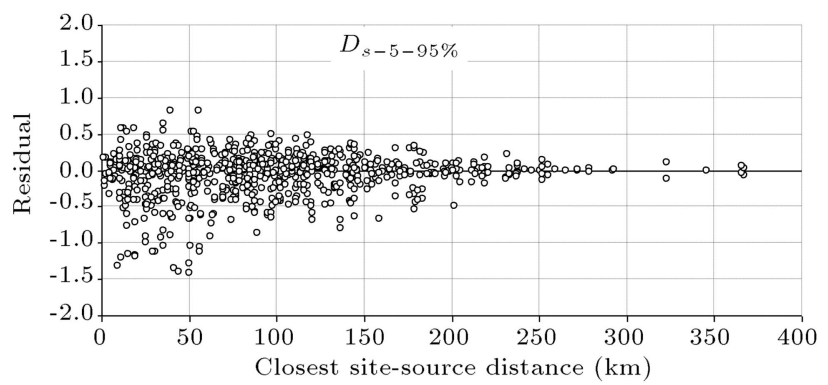

(a)

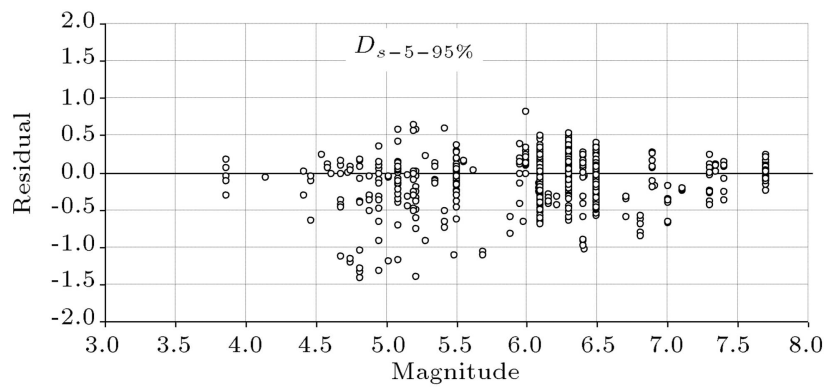

(b)

Figure 12. The distribution of residuals between the observed and predicted significant durations $\left(D_{s-5-95 \%}\right)$ for the proposed model with respect to (a) closest site-source distance and (b) magnitude.

tions in logarithmic units $(\ln ($ observed $)-\ln ($ predicted $))$ based on the predictive models for $D_{s-5-95 \%}$ and $D_{s-5-75 \%}$ against magnitude and distance measures. The spread of residuals in these figures represents the variability of individual data values, which could demonstrate the quality of a predictor. Residuals of the proposed models for $D_{s-5-95 \%}$ and $D_{s-5-75 \%}$ vary in the range of -1.5 to 1 showing less scattering than the predicted results of models of Bommer et al. [2]. The residuals for both definitions of significant duration do not show any trend with magnitude or distance, confirming that the fitting procedure is robust and appropriate. Similar conclusion was made through the work of Bommer et al. [2] when their suggested functional form was used in the analysis (see Figures 1 and 2 in [2]).

The scatter plot of residuals between the observed and predicted values against soil site conditions is illustrated in Figure 14. From inspection of this figure, the same prediction quality could be recognized for rock and soil sites.

A more comprehensive comparison made between different ground-motion duration prediction equations 


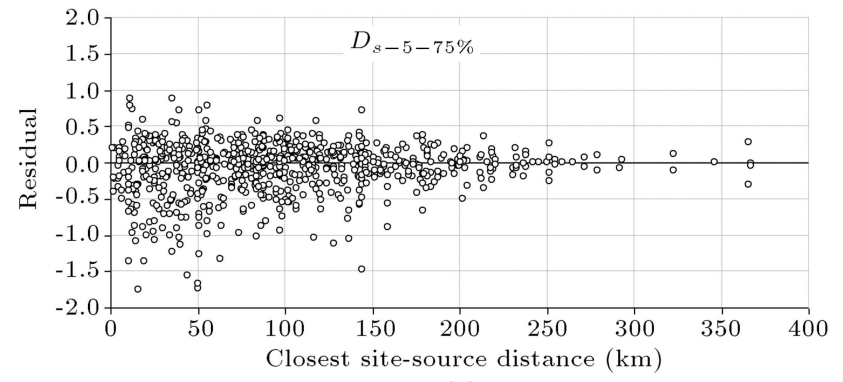

(a)

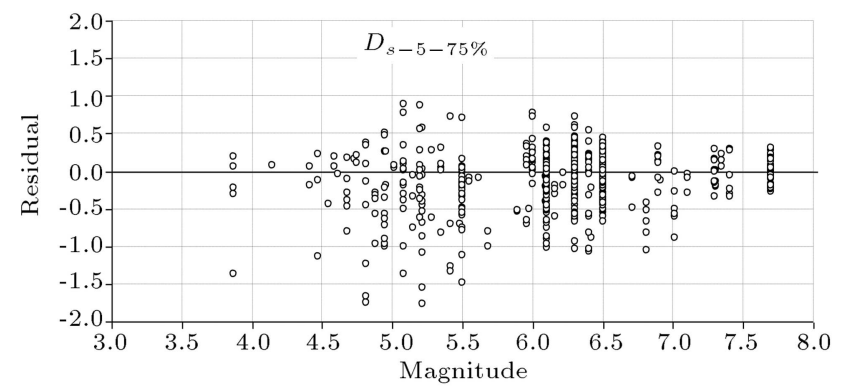

(b)

Figure 13. The distribution of residuals between the observed and predicted significant durations $\left(D_{s-5-95 \%}\right)$ for the proposed model with respect to (a) closest site-source distance and (b) magnitude.
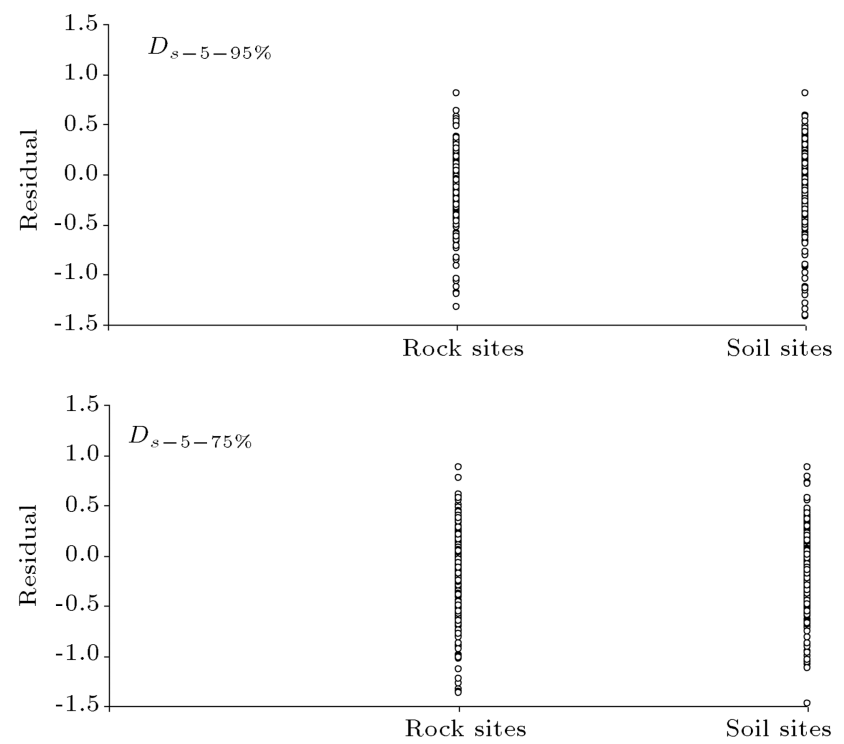

Figure 14. The distribution of residuals between the observed and predicted significant durations for the proposed model with respect to site conditions.

is shown in Figures 15 and 16 . In order to obtain an accurate evaluation and based on the validity of predicted values, models prepared in this paper have been re-examined with respect to the predictions in a specified distance (taken as $30 \mathrm{~km}$ ) at rock sites for different values of magnitude ranging from 4.5 to 8 . Results of the predicted values based on recently published empirical relationships by Bommer et al. [2] and Yaghmaei-Sabegh et al. [13] have been superim-

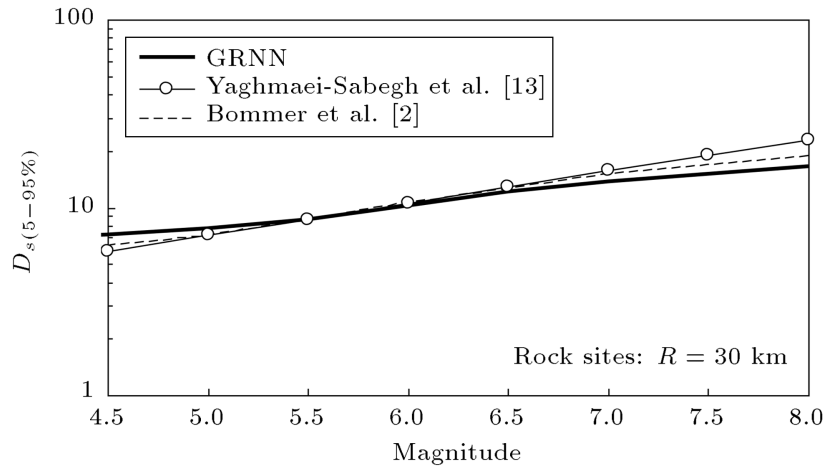

Figure 15. Comparison of the proposed GRNN model for significant duration $D_{s-5-95 \%}$ at a fixed distance measure $(R=30 \mathrm{~km})$ on rock sites.

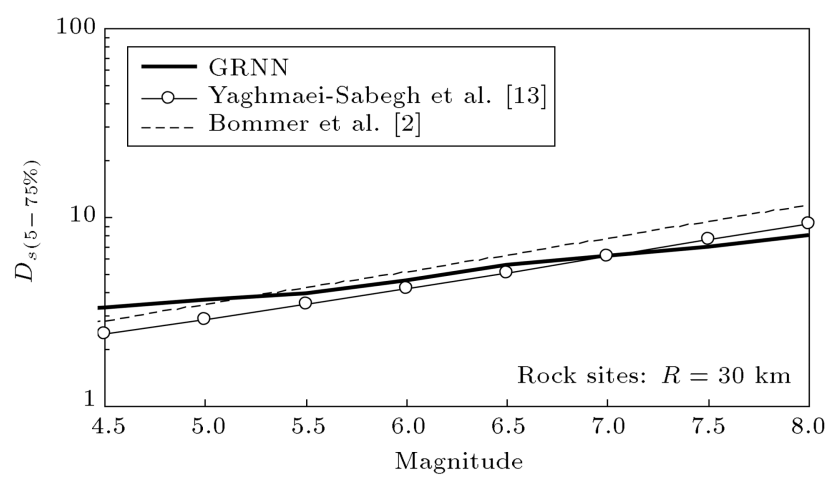

Figure 16. Comparison of the proposed GRNN model for significant duration $D_{s-5-75 \%}$ at a fixed distance measure $(R=30 \mathrm{~km})$ on rock sites.

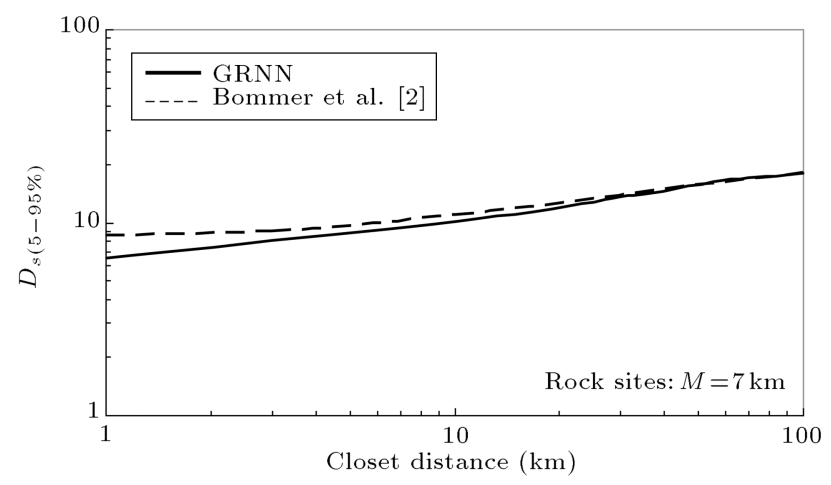

Figure 17(a). Variation of significant duration $D_{s-5-95 \%}$ in distance for moment magnitude $M_{w}=7$ at rock sites.

posed onto these figures. According to Figures 15 and 16, the suggested model is well matched with models of Bommer et al. [2] and Yaghmaei-Sabegh et al. [13], reconfirming the validity of ANN models designed in this paper. However, some discrepancy could be observed among three models which might be related to the database size and their different features. Plotting of the logarithmic scale has been adopted for these figures consistent with other publications in this path. Figure $17(\mathrm{a})$ shows the variation of significant 


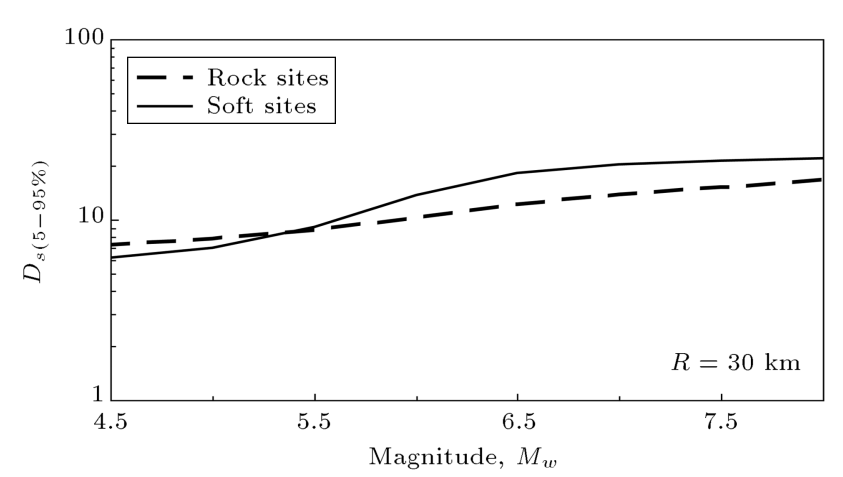

Figure 17(b). Variation of significant duration $D_{s-5-95 \%}$ in moment magnitude for rock and soft sites at a fixed distance measure $(R=30 \mathrm{~km})$.

duration $D_{s-5-95 \%}$ in distance for moment magnitude $M_{w}=7$ at rock sites based on the proposed model and predictions of Bommer et al. [2]. Good agreement between the models, particularly for distance larger than $10 \mathrm{~km}$ could be observed in this figure. Variation of significant duration $D_{s-5-95 \%}$ in moment magnitude for rock and soft sites at a fixed distance measure $(R=30 \mathrm{~km})$ is presented in Figure 17(b). This figure may possibly highlight soil effects on earthquake duration for strong earthquakes.

\section{Comparative analysis of GRNN and BP-MLFF models}

The prediction capability of Multi-Layer Feed-Forward (MLFF) network as the most popular ANN is evaluated in this section to show that there is a need for developing GRNN model. The structure of the implemented feedforward neural network is shown in Figure 18. As already discussed in Section 2 of the current paper, these types of neural networks were extensively applied in the past; however, finding the number of neurons forming hidden layers remains one of the unsolved tasks in the application of such networks. Neurons number in hidden layers, which controls the generalization capability of network, plays an important role in design of a MLFF. A single hidden layer, although with different neurons numbers, was used in the analysis to highlight this matter herein. The Kolmogorov's theorem $[59,60]$ could be considered as a simple rule

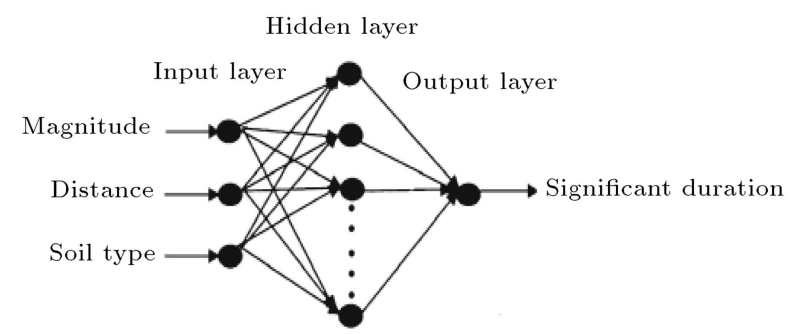

Figure 18. Architecture of MLFF network used in this study . (or initial guess) to recommend the neurons number of hidden layer (NHN):

$$
\mathrm{NHN}=2 \mathrm{NIN}+1
$$

where NIN represents the input neurons numbers. Thus, the neurons number in the hidden layer based on Kolmogorov's theorem was taken as $2 \times 3+1=7$, since there are three input neurons. Consequently, the training analysis started based on seven units in the hidden layer to learn the target mapping and continued by increasing neurons numbers to 9,12 , 15, and 18. A trial-and-error approach led to an optimal network architecture. The backpropagation (BP) learning scheme [61] which includes two phases of propagation and weight updating was adopted in this study as a common training technique in ANNs. The Levenberg-Marquardt training process as a variation of the Newton method was followed to train the designed BP-MLFF networks with different architectures. Weights were selected randomly for each training analysis. The tan-sigmoid function was adopted herein as an activation function of neurons in the hidden layer. Training and testing datasets were chosen similar to GRNN model. The predicted results of designed BP-MLFF models with different processing units are presented in Figures 19 and 20. Results contain the prediction of two common measures of significant duration: $D_{s-5-95 \%}$ and $D_{s-5-75 \%}$ separately. According to these figures, when there are few neurons (as the ANN configuration of 3-7-1), the network could not predict large values of significant duration well. Hence, the designed network does not model nonlinear features of function and the learning process may fail miserably. On the other side, with an increase in the neurons number of the hidden layer, the prediction capacity is improved; however, the network loses its ability to generalize.

Similar to GRNN models, the performance of ground-motion duration predictions resulting from training and testing data set is evaluated by the three indices: RMSE, $R$, and EF. Results are summarized in Tables 2 and 3 for $D_{s-5-95 \%}$ and $D_{s-5-75 \% \text {, re- }}$ spectively. The maximum values of EF and $R$ along with the lowest value of RMSE achieved based on the results of testing dataset show the higher generalization ability of networks with 7 and 9 neurons among others. Comparison of Tables 2 and 3 with Table 1 demonstrates higher performance of GRNN models obviously. This important result could be concluded based on the total three evaluation indices used in this paper. As an example, Efficiency Factor (EF) of GRNN model for prediction of $D_{s-5-95 \%}$ is 0.78 and 0.74 in training and testing datasets where the lower corresponding values of 0.53 and 0.51 are calculated for BP-MLFF networks in the best case. Root mean 

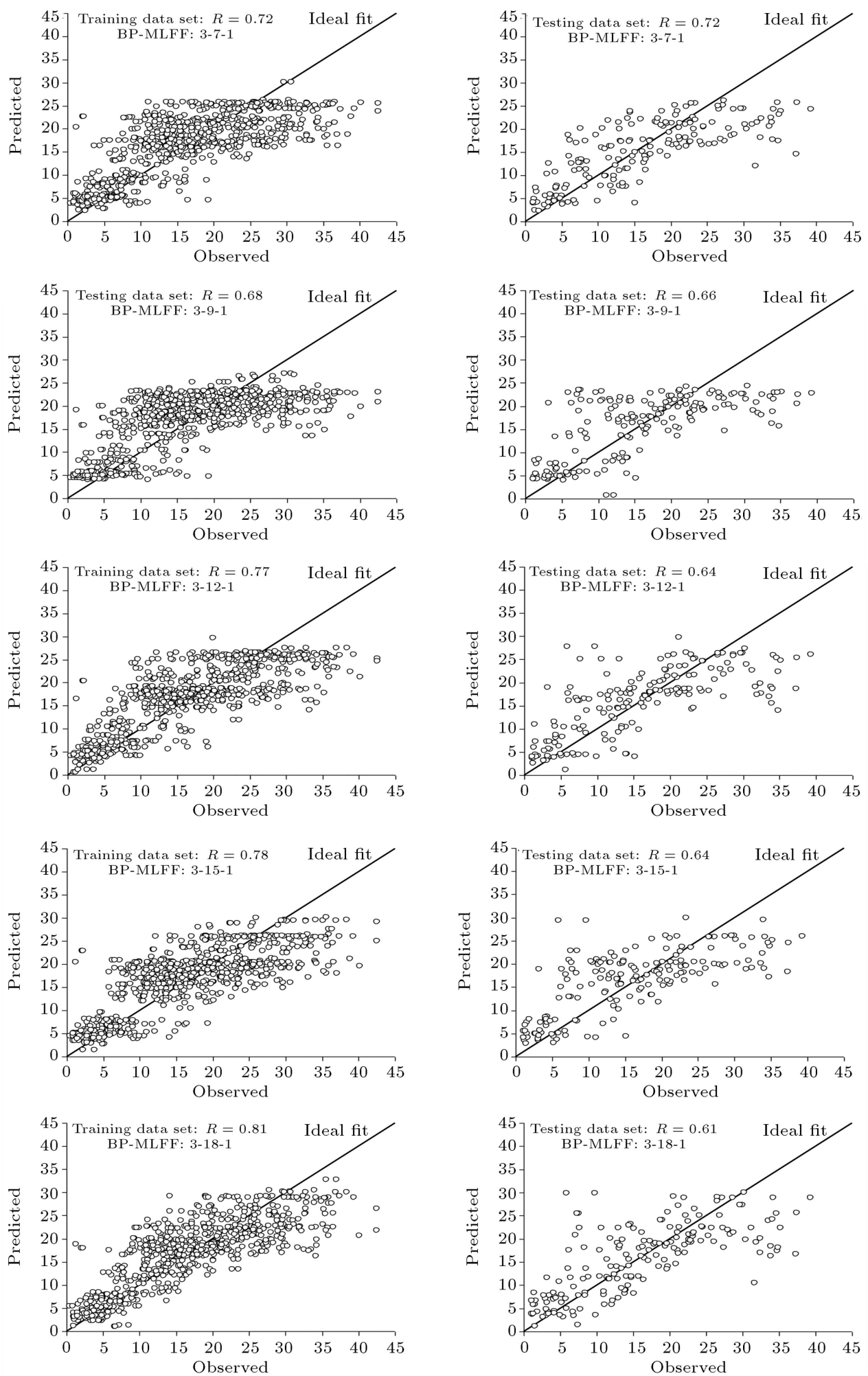

Figure 19. Observed values of $D_{s-5-95 \%}$ versus predicted values by BP-MLFF networks with different number of neurons in the hidden layer.

square error increases significantly when the BP-MLFF networks are applied to the prediction procedure. It is worth noting that the purpose of this section of paper is not to suggest a suitable structure for a BP-MLFF network. However, the results of BP-MLFF networks confirm that the prediction ability of such networks is very sensitive to the structure of designed network and is lower than GRNN performance. In this regard, designing and training of various networks to reach satisfactory results are required. As a result, due to the 

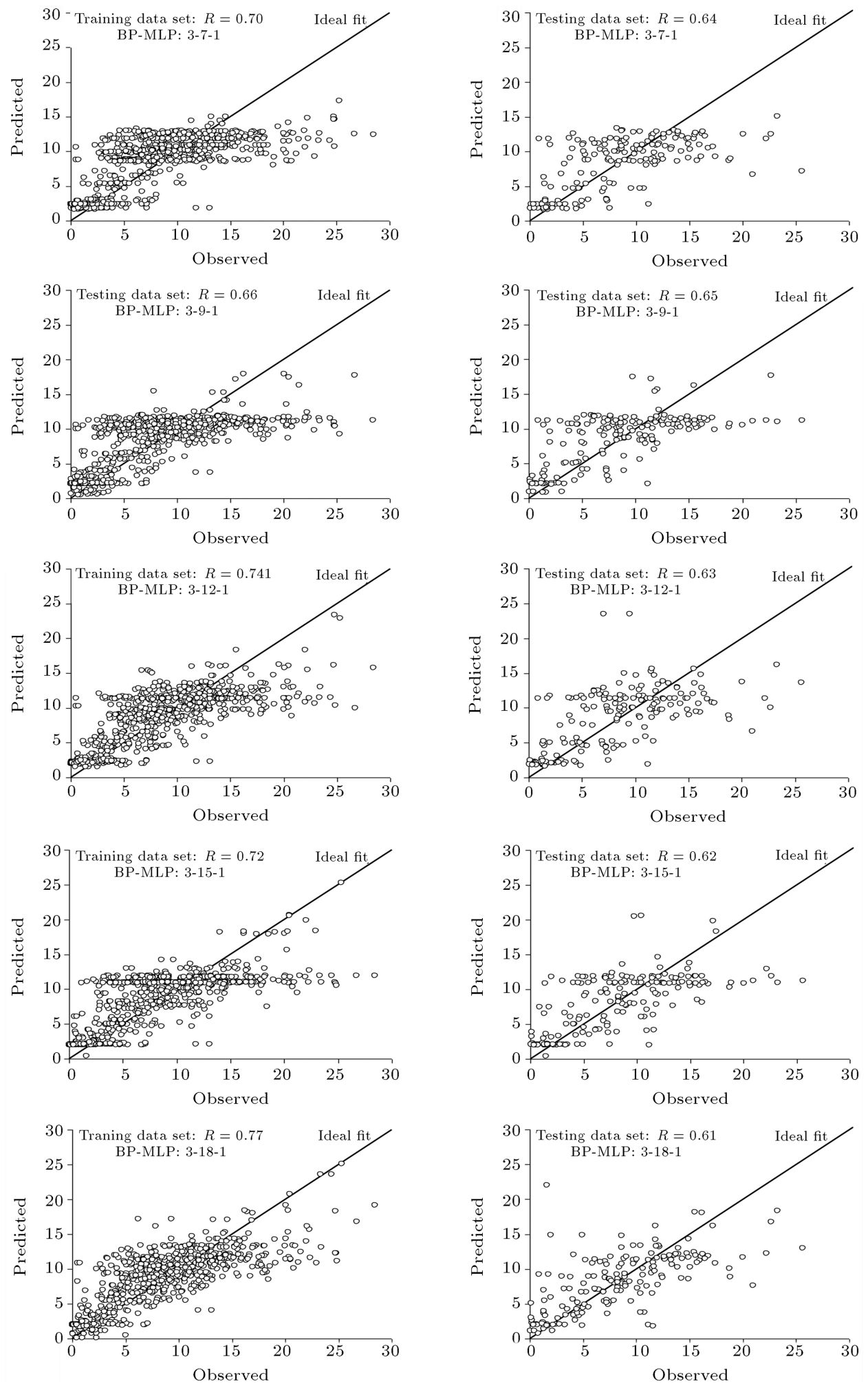

Figure 20. Observed values of $D_{s-5-75 \%}$ versus predicted values by BP-MLFF networks with different number of neurons in the hidden layer.

complex nature of earthquake ground-motion duration, prediction of such kinds of data with conventional BP-MLFF is difficult and requires special care where GRNN is a powerful technique that could resolve this problem simply.

\section{Summary and conclusions}

Improvement of predictive models, which are able to relate given ground motion characteristics to the seismological parameters, has been known to be a 
Table 2. Performance of designed BP-MLFF for predicting $D_{s-5-95 \%}$.

\begin{tabular}{|c|c|c|c|c|c|c|}
\hline \multirow{2}{*}{$\begin{array}{c}\text { BP-MLFF } \\
\text { topology }\end{array}$} & \multicolumn{3}{|c|}{ Training set } & \multicolumn{3}{|c|}{ Testing set } \\
\hline & $\boldsymbol{R}$ & $\mathbf{E F}$ & RMSE (sec) & $\boldsymbol{R}$ & $\mathbf{E F}$ & RMSE (sec) \\
\hline $3-7-1$ & 0.726 & 0.47 & 6.27 & 0.720 & 0.52 & 6.35 \\
\hline $3-9-1$ & 0.683 & 0.53 & 6.60 & 0.660 & 0.51 & 7.10 \\
\hline $3-12-1$ & 0.767 & 0.41 & 5.85 & 0.641 & 0.33 & 7.56 \\
\hline $3-15-1$ & 0.762 & 0.42 & 5.92 & 0.642 & 0.49 & 6.90 \\
\hline $3-18-1$ & 0.700 & 0.34 & 5.35 & 0.612 & 0.15 & 8.15 \\
\hline
\end{tabular}

Table 3. Performance of designed BP-MLFF for predicting $D_{s-5-75 \%}$.

\begin{tabular}{cccccccc}
\hline \multirow{2}{*}{$\begin{array}{c}\text { BP-MLFF } \\
\text { topology }\end{array}$} & \multicolumn{3}{c}{ Training set } & & \multicolumn{3}{c}{ Testing set } \\
\cline { 2 - 4 } \cline { 6 - 7 } \cline { 6 - 7 } & $\boldsymbol{R}$ & EF & RMSE (sec) & & $\boldsymbol{R}$ & EF & RMSE (sec) \\
\hline $3-7-1$ & 0.701 & 0.51 & 3.85 & & 0.640 & 0.50 & 4.20 \\
$3-1$ & 0.660 & 0.56 & 4.06 & & 0.650 & 0.49 & 4.10 \\
$3-15-1$ & 0.741 & 0.45 & 3.60 & & 0.620 & 0.35 & 4.40 \\
$3-18-1$ & 0.720 & 0.48 & 3.75 & & 0.620 & 0.33 & 4.42 \\
\hline
\end{tabular}

very imperative step in seismic hazard analysis. In this article, a new artificial network-based scheme was proposed to estimate earthquake record duration. A Generalized Regression Neural Network (GRNN) was implemented in the analysis and examined to predict a multi-faceted parameter of "earthquake ground motion-duration". Designed models were presented for two typical definitions of significant ground-motion duration defined based on 5-95\% and 5-75\% Arias Intensity $\left(D_{s-5-95 \%}\right.$ and $\left.D_{s-5-75 \%}\right)$. Different models were trained using the 950 ground motions recorded at active tectonic regions of Iran. Results of analysis showed good fitting with the training and testing records.

Unlike BP-MLFF network that needs too much convergence time, the time process of designed GRNN in this work is less than 5 seconds. It should be noted that the speed of convergence in nonlinear least square regression algorithm is dependent on the quality of an initial guess for the solution, which is not easy in all cases. The proposed method in this paper is able to remove the main shortcoming of the conventional method used to develop ground motion prediction (GMPEs) equations. The only parameter that needs to be selected for the general regression neural network is the smoothing parameter, playing a significant role in reaching an accurate prediction. Different values of this parameter were examined for general practical application of the proposed model. According to the analysis results, the smoothing parameter $\sigma=0.7$ was preferred to predict earthquake duration in the recommended models.
Based on the results of this paper, the easyto-use proposed GRNN model is found to be more effective in the prediction purpose of a complex parameter in seismology, ground-motion duration. This model could provide more accurate results than the models established based on BP-MLFF networks. The GRNN network gives the best generalization performance when RMSE takes 4.1, 2.68 values for two common measures of significant duration: $D_{s-5-95 \%}$ and $D_{s-5-75 \%}$, respectively, whereas the BP-MLFF network gives higher values of 6.35 and 4.1. Efficiency factor of GRNN model for prediction of $D_{s-5-95 \%}$ is 0.78 and 0.74 in training and testing datasets, respectively, where the lower corresponding values of 0.53 and 0.51 were calculated for BP-MLFF networks in the best case. Finding the number of neurons forming the hidden layers of MLFF networks remains as one of the unsolved tasks in the application of such networks. Therefore, designing and training of various networks to reach satisfactory results are required in most cases, particularly when the nature of data is complex. The comparative results of this article showed that the proposed GRNN model could solve such a problem simply and reduce the analysis time, too. Note that the proposed GRNN could resolve an unsuccessful prediction of BP-MLFF in long duration; however, the predicted duration with GRNN is underestimated for longer observation.

It is worth noting that the current paper was focused mainly on the capability of artificial neural networks; as for future works, a comparison of accuracy of the proposed method and other classical techniques 
as autoregressive integrated moving average (ARIMA) could be useful.

\section{Acknowledgement}

The contributions of BHRC are acknowledged to provide earthquake database of this study.

\section{References}

1. Bommer, J.J. and Martinez-Pereira, A. "The effective duration of earthquake strong motion", Journal of Earthquake Engineering, 3, pp. 127-172 (1999).

2. Bommer, J.J., Stafford, P.J., and Alarcón, J.A. "Empirical equations for the prediction of the significant, bracketed, and uniform duration of earthquake ground motion", Bull Seismol Soc Am, 99(6), pp. 3217-3233 (2009).

3. Kempton, J.J. and Stewart, P.J. "Prediction equations for significant duration of earthquake ground motions consideration site and near- source effects", Earthquake Spectra, 22, pp. 958-1013 (2006).

4. Reinoso, E. and Ordaz, M. "Duration of strong ground motion during Mexican earthquakes in terms of magnitude, distance to the rupture area and dominant site period", Earthquake Eng. Struct. Dyn., 3, pp. 653-673 (2001).

5. Iervolino, I., Manfredi, G., and Cosenza, E. "Ground motion duration effect on nonlinear seismic response", Earthquake Eng. Struct. Dyn., 35, pp. 21-38 (2006).

6. Nurtug, A. and Sucuoglu, H. "Prediction of seismic energy dissipation in SDOF systems", Earthquake Eng. Struct. Dyn., 24, pp. 1215-1222 (1995).

7. Hancock, J. and Bommer, J.J. "Using spectral matched records to explore the influence of strongmotion duration on inelastic structural response", Soil Dyn. Earthquake Eng., 27(4), pp. 291-299 (2007).

8. Trifunac, M.D. "Empirical criteria for liquefaction in sands via standard penetration tests and seismic wave energy", Soil Dyn. Earthquake Eng., 14(4), pp. 419426 (1995).

9. Youd, T.L. and Idriss, I.M. "Liquefaction resistance of soils: Summary report from the 1996 NCEER and 1998 NCEER/NSF workshops on evaluation of liquefaction resistance of soils", Journal of Geotechnical and Geoenvironmental Engineering, 127, pp. 297-313 (2001).

10. Rauch, A.F. and Martin, J.R. "EPOLLS model for predicting average displacements on lateral spreads", J. Geotech. Engrg, 126, pp. 360-371 (2000).

11. FEMA 1999, HAZUS99 Earthquake Loss Estimation Methodology: user's manual. Federal Emergency Management Agency, Washington DC (2003).

12. Whitman, R.V., Anagnos, T., Kircher, C., Lagorio, H.J., Lawson, R.S., and Schneider, P. "Development of a national earthquake loss estimation methodology", Earthquake Spectra, 13(4), pp. 643-661 (1997).
13. Yaghmaei-Sabegh, S., Shoghian, Z., and Sheikh, M.N. "A new model for the prediction of earthquake groundmotion duration in Iran", Nat. Hazards, 70, pp. 69-92 (2014).

14. Asencio-Cortés, G., Martínez-Álvarez, F., MoralesEsteban, A., and Reyes, J. "A sensitivity study of seismicity indicators in supervised learning to improve earthquake prediction", Knowledge-based Systems, 101, pp. 15-30 (2016).

15. Florido, E., Martínez-Álvarez, F., Morales-Esteban, A., Reyes, J., and Aznarte-Mellado, L. "Detecting precursory patterns to enhance earthquake prediction in Chile", Computers \& Geosciences, 76, pp. 112-120 (2015).

16. Yaghmaei-Sabegh, S. "A novel approach for classification of earthquake ground-motion records", Journal of Seismology, published online, 21(4), pp. 885-907 (2017).

17. Zhang, G.P. "Time series forecasting using a hybrid ARIMA and neural network model", Neurocomputing, 50, pp. 159-175 (2003).

18. McCulloch, W.S. and Pitts, W. "A logical calculation of the ideas immanent in nervous activity", Bull Mathematical Biophysics, 5, pp. 115-133 (1943).

19. Alippi, C., Polycarpou, M., Panayiotou, C., and Ellinas, G. "Artificial neural networks lecture notes in computer science", 19th International Conference, Limassol, Cyprus, September 14-17, Proceedings, Part I (2009).

20. Dysart, P.S. and Pulli, J.J. "Regional seismic event classification at the NORESS array: Seismological measurements and the use of trained neural networks", Bull. Seismol Soc. Am., 80(6B), pp. 1910-1933 (1990).

21. Dai, H. and MacBeth, C. "Application of backpropagation neural networks to identification of seismic arrival types", Physics of the Earth and Planetary Interiors, 101(3), pp. 177-188 (1997).

22. Xu, B., Wu, Z., Chen, G., and Yokoyama, K. "Direct identification of structural parameters from dynamics responses with neural networks", Engineering Applications of Artificial Intelligence, 17, pp. 931-943 (2004).

23. Chakraverty, S., Gupta, P., and Sharma, S. "Neural network-based simulation for response identification of two-storey shear building subject to earthquake motion", Neural Comput \& Applic, 19, pp. 367-375 (2010).

24. Kuźniar, K., Maciag, E., and Waszczyszyn, Z. "Computation of response spectra from mining tremors using neural networks", Soil Dyn. Earthquake Eng., 25, pp. 331-339 (2005).

25. Gentili, S. and Bragato, P. "A neural-tree-based system for automatic location of earthquakes in Northeastern Italy", Journal of Seismology, 10, pp. 73-89 (2006).

26. Asencio-Cortés, G. Martínez-Álvarez, F., Troncoso, A., and Morales-Esteban, A. "Medium-large earthquake magnitude prediction in Tokyo with artificial 
neural networks", Neural Comput. \& Applic., 28(5), pp. 1043-1055 (2017).

27. Kern, T. and Ting, S.B. "Neural network estimation of ground peak acceleration at stations along Taiwan high-speed rail system", Engineering Applications of Artificial Intelligence, 18, pp. 857-866 (2015).

28. Ahmad, I., El Naggar, M.H., and Naeem Khan, A. "Neural network based attenuation of strong motion peaks in Europe", Journal of earthquake Engineering, 12(5), pp. 663-680 (2008).

29. Arjun, C.R. and Kumar, A. "Neural network estimation of duration of strong ground motion using Japanese earthquake records", Soil Dyn. Earthquake Eng., 31, pp. 866-872 (2001).

30. Liu, Y., Ju, Y., Duan, C., and Zhao, X, "Structural diagnosis using neural network and feature fusion", Engineering Applications of Artificial Intelligence, 24, pp. 87-92 (2011).

31. Alarifi, A.S.N., Alarifi, N.S.N., and Al-Humidan, S. "Earthquakes magnitude predication using artificial neural network in northern Red Sea area", Journal of King Saud University-Science, 24, pp. 301-313 (2012).

32. Reyes, J., Morales-Esteban, A., and Martínez-Álvarez, F. "Neural networks to predict earthquakes in Chile", Applied Soft Computing, 13(2), pp. 1314-1328 (2013).

33. Martínez-Álvarez, F., Reyes, J., Morales-Esteban, A., and Rubio-Escudero, C. "Determining the best set of seismicity indicators to predict earthquakes, Two case studies: Chile and the Iberian Peninsula", KnowledgeBased Systems, 50, pp. 198-210 (2013).

34. Morales-Esteban, A., Martínez-Álvarez, F., and Reyes, J. "Earthquake prediction in seismogenic areas of the Iberian Peninsula based on computational intelligence", Tectonophysics, 59, pp. 121-134 (2013).

35. Panakkat, A. and Adeli, H. "Recurrent neural network for approximate earthquake time and location prediction using multiple sesimicity indicators", ComputerAided Civil and Infrastructure Engineering, 24, pp. 280-292 (2009).

36. Larran, P., Calvo, B., Santana, R., Bielza, C., Galdiano, J., Inza, I., Lozano, J.A., Armananzas, R., Santafe, G., Perez, A., and Robles, V. "Machine learning in bioinformatics", Brief Bioinform, 7(1), pp. 86-112 (2006).

37. Zamani, A., Sorbi, M., and Safavi, A. "Application of neural network and ANFIS model for earthquake occurrence in Iran", Earth Science Informatics, 6(2), pp. $71-85$ (2013).

38. Hagan, M.T., Demuth, H.B., and Beale M., Neural Network Design, PWS Publishing Company (1996).

39. Rajasekaran, S. and Amalraj, R. "Predictions of design parameters in civil engineering problems using SLNN with a single hidden RBF neuron", Computers and Structures, 80(31), pp. 2495-505 (2002).

40. Yen, G.G. "Identification and control of large structures using neural networks", Computers and Structures, 52(5), pp. 859-870 (1994).
41. Sunar, M., Gurain, A.M.A., and Mohande, M. "Substructural neural network controller", Computers and Structures, 78(4), pp. 575-581 (2000).

42. Zhang, A.H. and Zhang, L. "RBF neural networks for the prediction of building interference effects", Computers and Structures, 82(27), pp. 2333-2339 (2004).

43. Panakkat, A. and Adeli, H. "Neural network models for earthquake magnitude prediction using multiple seismicity indicators", International Journal of Neural Systems, 17(1), pp. 13-33 (2007).

44. Baddari, K., Aıfa, T., Djarfour, D., and Ferahtia, J. "Application of a radial basis function artificial neural network to seismic data inversion", Computers and Geosciences, 35, pp. 2338-2344 (2009).

45. Tang, C. "Radial basis function neural network models for peak stress and strain in plain concrete under triaxial stress", Journal of Materials in Civil Engineering, 22(9), pp. 923-934 (2010).

46. Specht, D.F. "A general regression neural network", IEEE Trans Neural Networks, 2(6), pp. 568-576 (1991).

47. Yaghmaei-Sabegh, S. and Tsang, H-H. "A new site classification approach based on neural networks", Soil Dyn. Earthquake Eng., 31, pp. 974-981 (2011).

48. Wasserman, P.D. "Advanced methods in neural network", Van Nostrand Reinhold, pp. 147-158 (1993).

49. Kim, B., Kim, S., and Kim, K. "Modelling of plasma etching using a generalized regression neural network", Vacuum, 71(4), pp. 497-503 (2003).

50. Tsoukalas, L.H. and Uhrig, R.E., Fuzzy and Neural Approached in Engineering, New York: Wiley (1997).

51. Kurup, P.U. and Griffin, E.P. "Prediction of soil composition from CPT data using general regression neural network", J. Computing in Civil Eng., 20(4), pp. 281-289 (2006).

52. Hanna, A.M., Ural, D., and Saygili, G. "Neural network model for liquefaction potential in soil deposits using Turkey and Taiwan earthquake data", Soil Dyn. Earthquake Eng., 27(6), pp. 521-540 (2007).

53. Yaghmaei-Sabegh, S. and Tsang, H-H. "Site class mapping based on earthquake ground motion data recorded by regional seismographic network", Nat. Hazards, 73, pp. 2067-2087 (2014).

54. Ambraseys, N.N. and Sarma, S.K. "Response of earth dams to strong earthquakes", Geotechnique, 17, pp. 181-213 (1967).

55. Page, R.A., Boore, D.M., Joyner, W.B., and Coulter H.W. "Ground motion values for use in seismic design of the trans-Alaska pipeline system", US Geological Survey Circular, 672 (1972).

56. Yaghmaei-Sabegh, S. and Lam, N.T.K. "Ground motion modelling in Tehran based on the stochastic method", Soil Dyn. Earthquake Eng., 30(7), pp. 525535 (2010). 
57. Building Seismic Safety Council (BSSC) "NEHRP recommended provisions for seismic regulations for new buildings and other structures developed for the federal emergency management agency", FEMA 302/303, Washington, D.C. (1997).

58. Guclu, D. and Dursum, S. "Artificial neural network modelling of a large-scale wastewater treatment plant operation", Bioprocess and Biosystems Engineering, 33(9), pp. 1051-1058 (2010).

59. Kolmogorov, A.N. "On the representation of continuous functions of several variables by superposition of continuous functions of one variable and addition", Doklady Akademii Nauk SSSR, 114, pp. 359-373 (1957).

60. Hecht-Nielsen, R. "Kolmogorov's mapping neural network existence theorem", in IEEE International Conference on Neural Networks, pp. 11-14 (1987).

61. Rummelhart, D.E., Hilton, G.E., and Williams, R.J.
"Learning internal representations by error propagation", Parallel Distributed Processing, 1, chapter 8, D.E. Rummelhart, and J.L. McCleland, Eds., Cambridge, MA (MIT Press) (1986).

\section{Biography}

Saman Yaghmaei-Sabegh obtained his PhD degree from Iran University of Science and Technology and was awarded the Elite Prize by the university in 2006 and 2007. He is currently an Associate Professor at The University of Tabriz and has been a Visiting Scholar at The University of Melbourne, Australia since 2007. He is a Member of the Iranian Earthquake Engineering Association. He has published more than 40 technical articles and has won award in the 14th International Conference on Earthquake Engineering in 2008 as a young researcher. 